\title{
Atuação do enfermeiro frente à criança e ao adolescente com câncer: limites e
}

\section{possibilidades}

\author{
Nurses' performance in relation to children and adolescents with cancer: limits and possibilities \\ El desempeño de las enfermeras en relación con niños y adolescentes con cáncer: límites y \\ posibilidades
}

Recebido: 19/10/2021 | Revisado: 27/10/2021 | Aceito: 02/11/2021 | Publicado: 04/11/2021

\author{
Bianka Queiroz da Silva \\ ORCID: https://orcid.org/0000-0003-2531-0665 \\ Instituto Nacional do Câncer, Brasil \\ E-mail: biankaqs@gmail.com \\ Michelly Cristynne Souza Bonifácio \\ ORCID: https://orcid.org/0000-0001-7771-6507 \\ Universidade Federal Fluminense, Brasil \\ E-mail: Michelly.bonifacio@live.com \\ Aline Cerqueira Santos Santana da Silva \\ ORCID: https://orcid.org/0000-0002-8119-3945 \\ Universidade Federal Fluminense, Brasil \\ E-mail: alinecer2014@gmail.com \\ Yasminn Canella Cabral Banjar Coelho \\ ORCID: https://orcid.org/0000-0001-7774-7573 \\ Universidade Federal Fluminense, Brasil \\ E-mail: yasminn.canella@ hotmail.com \\ Fernanda Garcia Bezerra Góes \\ ORCID: https://orcid.org/0000-0003-3894-3998 \\ Universidade Federal Fluminense, Brasil \\ E-mail: ferbezerra@gmail.com \\ Leila Leontina do Couto \\ ORCID: https://orcid.org/0000-0002-8948-5045 \\ Universidade Federal Fluminense, Brasil \\ E-mail: leila_leontina@hotmail.com \\ Rayssa Goulart Valente \\ ORCID: https://orcid.org/0000-0002-3532-3606 \\ Instituto Nacional do Câncer, Brasil \\ E-mail: rayssagoulart@ hotmail.com
}

\begin{abstract}
Resumo
Objetivo: analisar limites e possibilidades de atuação do enfermeiro na prestação da assistência à criança e ao adolescente com câncer. Metodologia: pesquisa qualitativa. Participaram 30 enfermeiros, a partir de entrevistas, seguindo roteiro semiestruturado, cujos dados foram processados no software IRAMUTEQ. Resultados: como possibilidade de atuação do enfermeiro na oncologia pediátrica verificou-se: a participação da família e da equipe multiprofissional no enfrentamento da doença da criança e do adolescente, e como limites: a falta de habilidades técnicas, de instrução familiar frente ao diagnóstico e do apoio psicológico institucional frente a morte. Conclusão: analisar limites e possibilidades da atuação do enfermeiro oncologista, se faz necessária, não apenas visando uma assistência de qualidade, mas por contribuir para uma visão ampliada, a partir do profissional, dando voz às suas emoções e sentimentos envolvidos na assistência oncológica.
\end{abstract}

Palavras-chave: Criança; Adolescente; Enfermagem oncológica; Assistência de enfermagem.

\begin{abstract}
Objective: to analyze the limits and possibilities of nurses' performance in providing care to children and adolescents with cancer. Methodology: qualitative research. Thirty nurses participated, from interviews, following a semistructured script, whose data were processed in the IRAMUTEQ software. Results: as a possibility for nurses to work in pediatric oncology, the following was verified: the participation of the family and the multiprofessional team in coping with the disease of children and adolescents, and as limits: the lack of technical skills, family instruction in the face of the diagnosis and institutional psychological support in the face of death. Conclusion: to analyze the limits and possibilities of the oncologist nurse's performance, it is necessary, not only aiming at quality assistance, but also for contributing to an enlarged view, from the professional, giving voice to his emotions and feelings involved in the oncological assistance.
\end{abstract}

Keywords: Child; Adolescent; Oncology nursing; Nursing care. 


\begin{abstract}
Resumen
Objetivo: analizar los límites y posibilidades del desempeño del enfermero en la atención a niños y adolescentes con cáncer. Metodología: investigación cualitativa. Participaron 30 enfermeras, a partir de entrevistas, siguiendo un guión semiestructurado, cuyos datos fueron procesados en el software IRAMUTEQ. Resultados: como posibilidad para el enfermero de trabajar en oncología pediátrica, se verificó lo siguiente: la participación de la familia y el equipo multiprofesional en el afrontamiento de la enfermedad de niños y adolescentes, y como límites: la falta de habilidades técnicas, instrucción familiar en el rostro del diagnóstico y el apoyo psicológico institucional ante la muerte. Conclusión: para analizar los límites y posibilidades del desempeño del enfermero oncólogo, es necesario, no solo con el objetivo de una asistencia de calidad, sino de contribuir a una visión ampliada, desde el profesional, dando voz a sus emociones y sentimientos involucrados en la asistencia oncológica.
\end{abstract}

Palabras clave: Niño; Adolescente; Enfermería Oncológica; Atención de Enfermería.

\title{
1. Introdução
}

O câncer se apresenta como a principal causa de morte por doença entre crianças e adolescentes de um a 19 anos de idade. De acordo com o Instituto Nacional de Câncer (INCA), para cada ano do triênio 2020/2022 de 8.460 novos casos serão diagnosticados. Todavia, apesar dos índices alarmantes, o câncer infanto-juvenil tem apresentado grande possibilidade de cura frente ao progresso no tratamento, correspondendo no aumento da sobrevida, em aproximadamente mais da metade dos casos. No Brasil, este índice perfaz cerca de $80 \%$ quando o diagnóstico ocorre em momento oportuno e tratados em centros especializados, possibilitando que a maioria deles tenha boa qualidade de vida após o tratamento adequado (INCA, 2021).

Contudo, apesar dos avanços no que tange o tratamento oncológico, o câncer ainda se apresenta como uma doença socialmente temida, na qual o diagnóstico remete ao medo da morte, trazendo consigo uma gama de sofrimentos que afeta não somente o indivíduo, mas toda a sua família. Dessa forma, uma criança diagnosticada com câncer altera toda a estrutura familiar, gerando conflitos e impactos causados pelos mitos da doença oncológica presente no imaginário social que influenciam negativamente na forma em que os envolvidos aceitarão essa doença (Rodrigues, Siqueira Junior, \& Siqueira, 2020).

Neste contexto, o enfermeiro é o profissional que estabelece uma relação interpessoal de ajuda, sendo o responsável por gerir o cuidado, estabelecer prioridades, e prover informações acerca do tratamento e os efeitos decorrentes deste, além de gerenciar a progressão da doença, impossibilidade de cura e terminalidade da vida, exigindo habilidades específicas diante das complexas necessidades apresentadas pelos pacientes e seus familiares. Desse modo, entende-se, que a atuação efetiva do enfermeiro frente aos pacientes oncológicos deve contemplar múltiplas ações, como o diagnóstico, o tratamento, a reabilitação além dos cuidados paliativos (Silva \& Bezerra, 2020).

Embora seja inegável os crescentes avanços na medicina, a atuação do enfermeiro na oncologia pediátrica ainda é cercada por grandes desafios, pois cuidar do público infanto juvenil, pode significar em muitos casos lidar rotineiramente com a morte, a incurabilidade e intensa pressão emocional vivenciada pela criança e seu familiar. Normalmente o câncer se apresenta como uma doença com prognóstico sombrio, onde a confirmação diagnóstica na idade infanto-juvenil provoca desgaste psíquico, sobretudo frente as internações, procedimentos invasivos por vezes dolorosos, além da radioterapia, quimioterapia, cirurgias e transplantes, gerando grande carga de sentimentos entre os envolvidos (Paula, Silva, Andrade, \& Paraiso, 2019).

Compreende-se, portanto, que a prática clínica na oncologia pediátrica impõem grandes dificuldades, uma vez que o processo de trabalho da enfermagem tornou-se mais complexo, pois exige uma equipe capaz de compreender as peculiaridades deste universo de cuidado. Onde se faz necessário profissionais capacitados, responsáveis, compromissados, com sensibilidade e empatia para realização de cuidado específico voltado à criança, ao adolescente e sua família. Neste sentido, o profissional enfermeiro se insere neste ambiente com uma postura humanística, pautado em uma qualificação com foco não somente sobre 
os aspectos da esfera técnico-científica, mas sobretudo os aspectos emocionais, como exigências da área oncológica (Santana, Costa, Corréa, \& Ximenes, 2017).

Desta maneira, frente os cuidados a serem prestados à criança e ao adolescente com câncer, deve considerar as diversas peculiaridades que permeiam este universo, sendo de extrema importância que o enfermeiro esteja seguro em suas práticas diárias e que ultrapasse seus limites técnicos ao cuidar deste segmento populacional em tratamento. À vista disto, torna-se indispensável que o enfermeiro busque ampliar o aprimoramento do cuidar, da responsabilidade e da sensibilidade para vivenciar as nuances do câncer infanto-juvenil, visto que, o processo de cuidar em oncologia pediátrica é desafiante, requerendo uma equipe de saúde atenta para o que medeia o universo infantil (Silva, Assis, \& Pinto, 2021).

Logo, a contínua exposição a dor, ao sofrimento, a terminalidade da vida, a expectativa do usuário e as limitações impostas pelo sistema de saúde influenciam mormente, o processo de trabalho da enfermagem na oncologia pediátrica revelando a necessidade de refletir e lidar com temores que se interpõem à sua atuação (Menin \& Petterson, 2015). Desse modo, pressupõem-se que o ato de cuidar neste cenário se reveste de grande complexidade, requerendo do enfermeiro o uso de estratégias que lhe possibilitem o enfrentamento desde a sobrecarga física à demanda psicológica intensa a qual é cotidianamente submetido na oncologia pediátrica (Souza et al.,2020). Nesta perspectiva, este trabalho, volta-se à compreensão de como esse profissional lida com os limites e possibilidades em atuar na oncologia pediátrica, visando contribuir não só para atenção e cuidado prestado, mas também a busca pelo bem-estar destes profissionais.

Neste sentido, o presente estudo objetiva analisar limites e possibilidades de atuação do enfermeiro na prestação da assistência à criança e ao adolescente com câncer.

\section{Metodologia}

Trata-se de um estudo descritivo exploratório de abordagem qualitativa, cuja elaboração foi pautada sob os Critério Consolidados de Relato de Pesquisa Qualitativa (COREQ). Foi realizado em duas unidades hospitalares públicas especializadas no diagnóstico e tratamento do câncer infanto-juvenil no estado do Rio de Janeiro-RJ, Brasil, denominadas de unidades A e B.

Os cenários de pesquisa foram elencados por se constituírem como unidades polo de atendimento à criança e ao adolescente com câncer, possibilitando investigar a atuação do enfermeiro oncologista. Essas unidades têm como perfil: a) alta especificidade técnica no que tange o diagnóstico e tratamento; b) unidades de maior tempo de permanência do paciente para tratamento; c) incorporação de tecnologias de alta densidade para tratamento das crianças e adolescentes gravemente enfermos; d) e por dispor de Centro de Terapia Intensiva Pediátrica (CTIP), setor de quimioterapia e radioterapia, além de amplo centro cirúrgico e acompanhamento ambulatorial. Destaca-se que os participantes do estudo atuavam no CTIP, setor de quimioterapia e Unidade de internação.

Foi incluído na pesquisa apenas o profissional enfermeiro atuante nos setores supracitados por, no mínimo, seis meses visando que o participante estivesse adaptado e compreendesse o processo de trabalho nos cenários em questão, independentemente do tempo de formação em enfermagem. Como critérios de exclusão- enfermeiro que desenvolvesse atividades apenas de cunho administrativo ou os que estivessem de licença de qualquer tipo no período de coleta de dados. A respeito da amostra, os participantes foram selecionados por conveniência durante o período de coleta de dados, de agosto a outubro de 2019, dessa forma, o número amostral final, foram trinta enfermeiros, destaca-se que do total de profissionais convidados para participar da pesquisa, oito encontravam-se de férias, sendo dois na instituição A e os demais na instituição B.

O levantamento de dados ocorreu por meio de entrevistas face a face, que seguiu um roteiro semiestruturado elaborado pelos pesquisadores, dividido em duas partes, onde a primeira continha questões fechadas para a caracterização do perfil dos participantes da pesquisa, como: sexo, idade, escolaridade, tempo de formação, tempo de atuação em enfermagem, 
tempo de atuação em oncologia pediátrica, se possuía curso de especialização Latu ou Stricto Sensu e/ou curso na área de oncologia.

A segunda parte contou com perguntas abertas referente à atuação do enfermeiro frente à criança e adolescente com doença oncológica, a saber: como começou a trabalhar com oncologia pediátrica? Quais os limites e possibilidades encontrados na prestação da assistência frente à criança e ao adolescente com câncer? Qual foi a maior dificuldade vivenciada na assistência prestada frente à criança e ao adolescente com câncer?

O convite aos enfermeiros atuantes no setor deu-se através do contato prévio da enfermeira que exerce a função de gerente do setor em cada unidade, que disponibilizou o total de enfermeiros, a escala de cada um e o melhor horário para realização da entrevista. A entrevista foi realizada por um dos pesquisadores e uma sala reservada com apenas o entrevistador e o participante a fim de garantir a privacidade. A coleta de dados ocorreu na data previamente agendada, onde o pesquisador apresentou-se ao participante e explicou de forma detalhada e objetiva o que concernia a pesquisa e os seus objetivos.

A entrevista teve duração média de 30 minutos, onde foram gravadas em mídia digital (apenas áudio) e, depois transcritas. Em respeito ao anonimato e sigilo os participantes foram identificados pelo uso de códigos alfanumérico (ENF 1, ENF 2...), na sequência em que as entrevistas foram realizadas. Nenhuma entrevista teve que ser repetida. Ressalta-se que, o roteiro utilizado para o levantamento de dados foi previamente testado qualitativamente por três pesquisadores da equipe sem necessidade de adequações.

O processamento dos dados ocorreu a partir do uso do software Interface de $\mathrm{R}$ pour Analyses Multidimensionnelles de Textes Et de Questionnaires (IRAMUTEQ), mediante aos métodos Nuvem de Palavras e Classificação Hierárquica Descendente (CHD). Para tanto, foi necessário preparar o corpus textual de acordo com o estabelecido para análise pelo software IRaMuTeQ. Desse modo, o corpus foi revisado, onde os acentos foram retirados, as siglas uniformizadas, palavras compostas foram unidas através do uso de underline, além da correção de pontuação, ortografia e digitação. Após a transcrição e os ajustes descritos realizada no LibreOffice Writer do pacote LibreOffice.org, o arquivo foi salvo como documento de texto que usa codificação de caracteres no padrão UTF-8 (Unicode Transformation Format 8 bit codeunits) (Souza, Wall, Thueler, Lowen, \& Peres, 2018).

A interpretação dos dados ocorreu segundo os pressupostos da Análise Temática, que busca encontrar os núcleos de sentido que compõem uma comunicação, o que leva à compreensão do objeto analítico (Minayo, 2014). Destaca-se que para a interpretação dos dados, a partir da CHD, foram resgatadas as formas ativas (substantivos, adjetivos, advérbios e formas não reconhecidos) de cada classe de segmentos de texto, com destaque para as que obtiveram no teste qui-quadrado ( $\chi^{2}$ ) um valor $\geq 3,84$ e um p-valor $<0,0001$, que revela a altíssima significância na associação das palavras (léxicos) na sua respectiva classe.

Os aspectos éticos foram contemplados de acordo com a Resolução n 466 de 2012 do Conselho Nacional de Saúde, e todos os participantes assegurados sobre o sigilo, anonimato e confidencialidade de suas informações, mediante o aceite do TCLE. O estudo teve seu parecer aprovado sob o n³.113.786 e CAAE: 93546418.6.3001.5274.

\section{Resultados}

Participaram do estudo 30 (100\%) enfermeiros que atuavam nas duas unidades em diferentes turnos, dos quais 24 (80\%) eram do sexo feminino. A média de idade foi de 42 anos, sendo mais novo com 32 anos e o mais velho com 62 anos de idade. Dentre os participantes pesquisados, 22 (73,3\%) possuíam curso de especialização Latu Sensu em oncologia e 7 (23,3\%) tinham como titulação mais avançada, o curso de mestrado. Foi evidenciado um perfil de enfermeiros com tempo médio de formação acima de 10 anos. Dentre os participantes 17 (56,6\%) atuavam há mais de dez anos em oncologia pediátrica. Com relação à realização de curso de capacitação em oncologia, 17 (56,6\%) enfermeiros disseram ter realizado. 
Após análise realizada pelo software, pela estatística básica, o corpus textual foi formado por 30 textos, correspondente ao número exato de formulários respondidos, e 16136 palavras, dentre essas, 2125 formas distintas e 1069 hápax (única ocorrência).

Inicialmente, visando identificar os limites e possibilidades de atuação do enfermeiro frente à criança e ao adolescente com câncer, adotou-se o método Nuvem de Palavras, que permite o agrupamento e organização gráfica das palavras em consonância com sua frequência de aparecimento, possibilitando identificar palavras-chave de maior recorrência no corpus textual através da análise lexical simples. Assim, cada palavra tem o tamanho proporcional a sua frequência, facilitando identificar os termos que foram preponderantes no conjunto de termos analisados, como mostra a Figura 1.

Figura 1 - Nuvem de Palavras. Rio de Janeiro, RJ, Brasil, 2020.

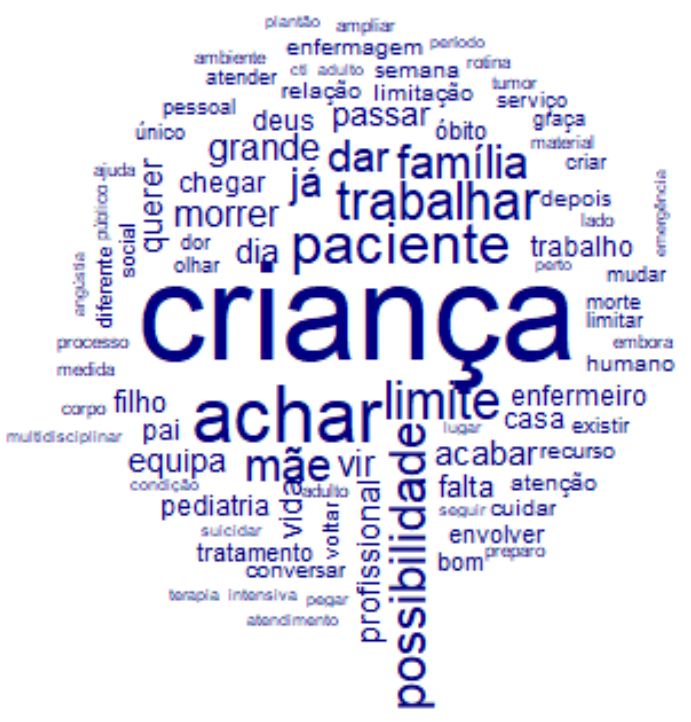

Fonte: Software IRAMUTEQ (2020).

Para interpretar a Nuvem de Palavras foram escolhidos os termos com maior frequência, sendo possível observar vocábulos centrais e recorrentes que possibilitaram identificar os limites e possiblidades de atuação do enfermeiro frente à criança e ao adolescente com doença oncológica, a saber: criança (206), achar (106), paciente (71), trabalhar (65), limite (64), possibilidade (60), família (52), mãe (52), dar (50) grande (39), morrer (38), profissional (30), vida (30), equipe (29), pediatria (24), trabalho (24), Deus (21), tratamento (20), óbito (18), dentre outras, as quais serão detalhadas posteriormente.

$\mathrm{Na}$ segmentação do corpus textual por classes de segmentos de texto e seus vocábulos, através da Classificação Hierárquica Descendente (CHD), foram destacadas as ideias centrais resultantes das respostas dos profissionais entrevistados. Assim, a análise hierárquica reteve 454 segmentos de texto, classificando 373 deles, logo, obteve um aproveitamento de $82,16 \%$. Dessa forma, formou-se dois subcorpos com quatro classes estáveis, ou seja, quatro agrupamentos de unidades de segmentos de texto com termos semelhantes e que possuem ligações entre si. A partir da análise, os dados foram organizados em um dendrograma (Figura 2) que expõe as relações entre as classes e o percentual de cada uma delas em relação ao total do corpus analisado. 
Figura 2- Dendograma da Classificação Hierárquica Descendente. Rio de Janeiro, RJ, Brasil, 2020.
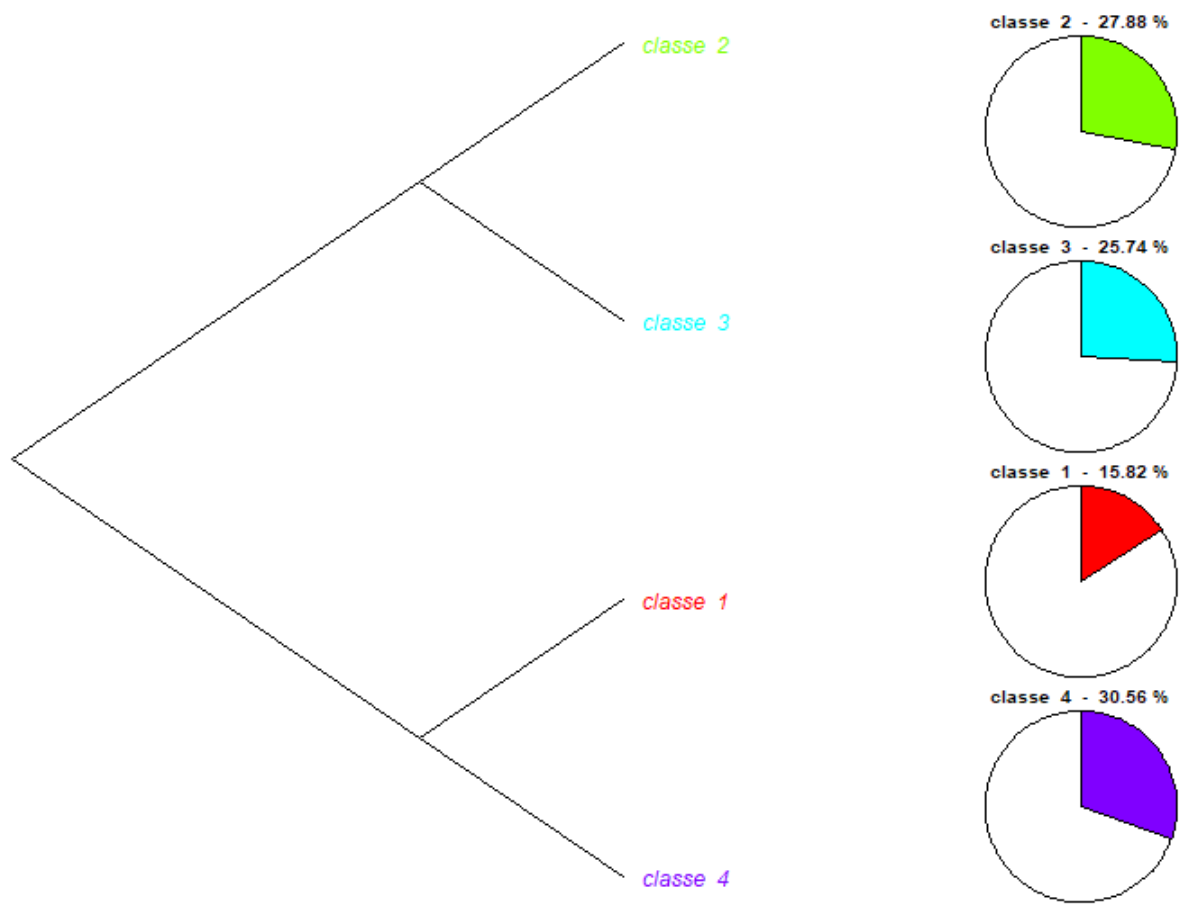

Fonte: Software IRAMUTEQ (2020).

No dendograma, o corpus textual dividiu-se em dois subcorpus, sendo o primeiro composto por uma subdivisão com duas partes, a saber, a Classe 4 em roxo (30,6\%), e a classe 1 em vermelho (15,8\%). E o segundo, composto por duas partes a classe 2 em verde $(27,9 \%)$ e a Classe 3 em azul (25,7\%), que apresentam associação entre si, porém distintas. Frente a análise do corpus pelo software foi possível compreender que as Classes 1 e 4 estão associadas entre si, porém opostas em termos lexicais.

As classes foram analisadas minuciosamente a fim de compreender seus significados através dos fragmentos textuais, e assim, denominar cada uma delas, quais sejam: Classe 1: A família, o conhecimento, habilidades específicas e a equipe multidisciplinar como limites e possibilidades de atuação da enfermagem; Classe 2: Atuação da equipe de enfermagem diante da família frente ao processo de morte e morrer na oncologia pediátrica; Classe 3: O uso de estratégias pela enfermagem na atuação frente à criança e adolescente com câncer; e Classe 4 - limites e possibilidades da atuação do enfermeiro na oncologia pediátrica.

Classe 1- A família, o conhecimento, habilidades específicas e a equipe multidisciplinar como limites e possibilidades de atuação do enfermeiro.

A classe 1 apresenta 15,8\% dos segmentos de texto, na qual as formas ativas que apresentaram Qui ${ }^{2} \geq 3,84$ e valor de $\mathrm{P}$ $<0.0001$ em ordem decrescente foram: família, enfermeiro, equipe, enfermagem, multidisciplinar, social, cuidar, preparo, corpo, ampliar, existir, olhar, paciente, terapia intensiva, assistente, processo e medida.

Família foi um termo prontamente enunciado pelos enfermeiros, como grande possibilidade para sua atuação, por ser reconhecida como parte fundamental no processo de adoecimento e de cuidar da criança com câncer, além disso a família possibilita a melhoria do diálogo com a equipe. 
A gente sempre tem possibilidades de melhoria, de afinamento desse diálogo, entre a família, equipe e a assistência para a gente ter possibilidades futuras melhores e mais concretas. (ENF 09)

É importante que a gente faça o melhor no sentido de estar junto, de integrar e interagir com essa família, essa criança, considerando que a família é fundamental nesse processo. (ENF 19)

Eu vejo que deveria ter mais trabalhos com a família, quando a gente busca sobre isso a gente não vê muito isso aqui nesta instituição. (ENF 03)

Ainda nesta compreensão, a partir do agrupamento de fragmentos textuais, foi possível observar alguns limites que se entrelaça na atuação do enfermeiro no que tange a díade criança-família no cenário da oncologia pediátrica.

As mães e as próprias crianças compartilham muitas dificuldades, muitas necessidades, muitos medos com a gente e aí a gente acaba se envolvendo com essas crianças, [...] o primeiro óbito para mim foi bastante difícil por conta desse envolvimento. (ENF 22)

Quando essa criança que estava em tratamento, durante muitos anos com a gente vai a óbito, para gente é muito ruim porque as vezes a gente é a única pessoa próxima da mãe e da criança, então cria um vínculo muito grande com elas. (ENF 08)

Integrar e interagir com essa família, essa criança, considerando que a família é fundamental nesse processo, e a gente tem que considerar essa família como parte da criança, então quando a criança adoece a família adoece junto com ela. (ENF 19)

Evidenciou-se pelas repostas que os profissionais de enfermagem compreendem que a participação da equipe multidisciplinar no cenário da oncologia pediátrica, corresponderia em grandes possibilidades para sua atuação no enfrentamento da doença diante da família e a criança, sobretudo nos casos mais graves e de terminalidade.

A maior limitação da enfermagem apresenta é não ter no tempo certo toda a equipe multidisciplinar, não só o médico, o enfermeiro, mas um assistente social, psicólogo, todos aqui dentro, acho que esse é um limite de atuação para gente. (ENF 24)

É com a equipe multidisciplinar que a gente sempre tem possibilidades de melhoria desse diálogo entre a família e a equipe, além de ampliar bastante as possibilidades da assistência ao paciente. (ENF 09)

Um momento muito próximo da terminalidade, então não tem como preparar bem essa família, eu acho que assim, muitas vezes, por não haver outros profissionais ao seu lado nesse momento, é ruim. (ENF 02)

Ainda nesta classe a falta de conhecimento e habilidades específicas foi associada pelos enfermeiros como fator limitante para sua atuação neste cenário da oncologia pediátrica, sobretudo frente ao cuidado paliativo e o processo de terminalidade.

E eu acho que o grande limitador de trabalhar com uma criança em oncologia é você realmente não conhecer as doenças, não conhecer os protocolos, para você trabalhar você tem que ter um amplo conhecimento das doenças e dos protocolos que você vai administrar. (ENF 06)

Eu acho que a grande dificuldade que nós ainda temos é em relação ao cuidado paliativo, porque na prática é bem diferente da teoria. E isso muitas vezes é ruim, para gente, precisamos entender esse processo. (ENF 02)

Conhecer a evolução da doença, estadiamento e as diferenciações entre cada uma, o porquê de ela estar apresentando isso naquele momento? Tendo sinais e sintomas específicos, interação medicamentosa, quimioterapia, muitos profissionais não conhecem e ficam sofrendo com isso. (ENF10)

\section{Classe 2 - Atuação do enfermeiro diante da família frente ao processo de morte e morrer na oncologia pediátrica.}

A classe 2 apresenta 27, 8\% dos segmentos de texto, na qual as formas ativas que apresentaram Qui ${ }^{2} \geq 3,84$ e valor de $\mathrm{P}<0.0001$ em ordem decrescente foram: mãe, pai, tratamento, adulto, diferente, dor, óbito, mudar e filho. 
Para cuidar das crianças com câncer e seus familiares, a equipe de enfermagem relata alguns fatores que limitam a sua atuação frente aos estágios do processo de morte e morrer, pois o cuidado é diferenciado, e, portanto, difícil, tendo em vista os aspectos operacionais e relacionais com a família, sobretudo com a mulher mãe.

O ponto mais dificil, é a "passagem" (morte) da criança e do adolescente ao lado dos pais, principalmente da mãe porque a gente vê a figura feminina muito presente, a mãe muito presente principalmente nas internações. (ENF 17)

Eu enquanto mãe, nunca que eu gostaria que uma filha minha estivesse passando por uma situação daquela, então a gente se coloca no lugar e sofre junto. (ENF 15)

Eu não queria que isso acontecesse no meu plantão, mas aconteceu. E essa mãe só gritava o meu nome, porque ela criou um vínculo comigo, e o que eu podia fazer era abraçar essa mãe, chorei muito, ela falava meu nome, gritava e chorava eu abracei e acolhi, falei algumas palavras para tentar confortar. (ENF 16)

Os profissionais de enfermagem apontaram a falta de informação, de instrução e de preparo psicológico dos pais acerca do tratamento de seus filhos como um fator limitante de sua atuação e possibilidade de cura da criança.

Eu acho que o ganho maior para gente é que os pais entendam e não banalizem o tratamento, nem a doença, porque não entende a dimensão, e às vezes a gente quer explicar e fica pensando será que vai entender? Eles precisam estar esclarecidos da importância de trazer a criança, de manter o tratamento. (ENF 04)

Às vezes a família não tem nível de instrução, não sabe ler e escrever e a gente tem que parar para fazer orientação de alta, porque o cuidado domiciliar é fundamental para a criança ter sucesso no tratamento, porque ela pode morrer por uma complicação infecciosa e não concluir o tratamento. (ENF 09)

Quando a família não é bem conduzida lá fora, e esse paciente entra, e é intubado aqui no CTI e realiza vários procedimentos invasivos e eles decidem tardiamente fechar as possibilidades terapêuticas, isso para mim é muito complicado. (ENF 14)

\section{Classe 3 - $O$ uso de estratégias defensivas pelo enfermeiro na oncologia pediátrica}

A classe 3 apresenta 25,7\% dos segmentos de texto, na qual as formas ativas que apresentaram $\mathrm{Qui}^{2} \geq 3,84$ e valor de $\mathrm{P}$ $<0,0001$ em ordem decrescente foram: deus, querer, graça, dia, semana e embora.

Esta classe destacou de forma relevante o desgaste físico e emocional vivenciado pela enfermagem na prestação dos cuidados à criança, adolescente e sua família frente ao processo de morrer, sendo reconhecido por eles como um limite de sua atuação neste cenário, onde muitos adotam estratégias defensivas, como o afastamento dos familiares, além do uso da espiritualidade e a religiosidade como enfrentamento diante dessa situação.

No caso de óbito eu fico assim, mais restrita e evito contato com os familiares que a gente tem mais vínculo, pois é o momento que o pai e a mãe precisam mais de apoio. (ENF 16)

Eu prefiro não estar presente, quando eu sei que aquela criança está em fim de vida, eu falo assim: meu Deus, tomara que não seja no meu dia, tomara que eu não esteja presente, mas se acontece eu tento me prevalecer de forças e de Deus para estar ali presente. (ENF 16)

Eu criei vínculo, eu costumo tentar não criar vínculo, é um mecanismo de defesa. (ENF 01)

A falta de apoio psicológico e de preparo específico para lidar com a situação de morte do paciente, foi apontado pelos enfermeiros como fator limitante de sua atuação na oncologia pediátrica, visto que o câncer se apresenta como uma grave doença, com tratamentos severos que podem levar a criança e ao adolescente à morte.

A maior dificuldade continua a mesma de todo mundo, o suporte psicológico que a gente não tem, isso é fundamental, e a gente não tem nenhum apoio em nada. (ENF 12)

Eu acho assim, que os profissionais deveriam ter um acompanhamento psicológico, é uma barra para nós, a gente tenta não se envolver, mas não consegue, não tem como ser diferente. (ENF 16) 
Limites para cuidar das crianças em fase terminal, não tem jeito. Esse é meu limite, e você sabe que também é o limite da criança e da família [...] seria um limite assim, mais psicológico. (ENF 30)

\section{Classe 4 - A falta de recurso humano, físico e de treinamento em serviço como limite de atuação do enfermeiro na oncologia pediátrica.}

A classe 4 apresenta 30,6\% dos segmentos de texto, na qual as formas ativas que apresentaram Qui ${ }^{2} \geq 3,84$ em ordem decrescente foram: achar, profissional, bom, limite, relação, humano, recurso.

Nesta classe, os enfermeiros pontuam como limitação para atuação na oncologia pediátrica, a falta de recurso humano e material frente à criança, ao adolescente e a família, onde a carência de insumos impostas pelo sistema prejudicam a condução da assistência.

Atualmente o hospital está passando por uma fase muito difícil de insumos, a gente está com problemas, um dia é o raio x que não está funcionando, outro dia não tem cateter. (ENF 21)

Eu acho que o que limita muito o cuidado hoje em dia aqui nesta instituição é a questão dos recursos humanos [...] isso limita porque a gente gostaria de ter mais tempo para prestar uma melhor assistência. (ENF 02)

Bom a gente trabalha em serviço público, né? Então as vezes é a questão de não ter uma droga, uma quimioterapia e ter que alterar protocolo porque não tem aquela droga, isso é realmente bem triste. (ENF 09)

Ainda sobre esta vertente, os enfermeiros relataram como limite de atuação na oncologia pediátrica, a falta de literatura, palestras em serviço e a implementação da educação permanente, para a prestação de uma assistência qualificada e assertiva.

Eu sinto falta, por exemplo de ter palestras voltadas para enfermagem sobre oncologia, como uma ação setorial. (ENF 25)

Eu acho que o que falta, seria mais cursos, palestras para a gente ter estudo, ter mais conhecimento, acho que é o que falta. (ENF 25)

A gente deveria ter mesmo uma educação permanente por estar trabalhando com essas crianças, até porque são patologias que mudam, muitas pesquisas mudam continuamente o tratamento, acho que é isso, que falta isso para gente aqui. (ENF 27)

\section{Discussão}

Compreende-se, a partir dos achados deste estudo, que apesar da vasta produção bibliográfica sobre o câncer, muitos desafios inerentes à doença oncológica ainda persistem e são enfrentados cotidianamente pelos enfermeiros, sobretudo os enfermeiros da oncologia pediátrica. Apesar da grande maioria dos enfermeiros do estudo apresentarem uma média de atuação na área oncológica pediátrica em torno de dez anos, as dificuldades relatadas ainda estão associadas as questões psicológicas relacionadas à finitude/morte de uma criança ou adolescente e ao sofrimento vivido na rotina desta clínica por todos os envolvidos.

Frente aos achados ora encontrados, constatou-se como possibilidade de sua atuação, a participação da família, relatado por eles como elo fundamental no processo de adoecimento dos filhos, articulada com a participação da equipe multidisciplinar no enfrentamento da doença oncológica. Outro destaque dado pelos participantes, foi com relação ao desconhecimento e habilidades técnicas necessárias para atuar na oncologia pediátrica, bem como a relação estabelecida com a criança e sua família durante a hospitalização, além da falta de preparo psicológico vivenciado pelos familiares e enfermeiros frente a morte.

A participação da família, foi destacada como parte fundamental no processo de cuidar da criança e do adolescente com câncer. O que torna necessário o estabelecimento de uma relação amistosa com a criança e sua família, durante o processo 
de internação, possibilitando a criação de vínculos e a ampliação do diálogo entre as partes. Entendendo, portanto, que a entrada do acompanhante no hospital, abriu a possibilidade de um cuidado relacional e social, de modo que não se pode mais cuidar de um sem o outro, pois a criança e a família tornaram-se um só cliente (Leite et al., 2020).

No entanto, a falta de preparo psicológico e de instrução dos familiares frente ao diagnóstico de câncer e tratamento, por vezes se apresenta como limite de atuação dos enfermeiros. Diante disso, foi possível observar que os enfermeiros não consideram apenas o bem-estar físico do cliente, mas também levam em conta o familiar, pois percebem as necessidades apresentadas por eles, exigindo um cuidado integral e ao mesmo tempo singular, que vai para além da execução de procedimentos técnicos (Markus et al., 2017).

Disso decorre a necessidade de a equipe estar apta a identificar sentimentos de medo, tristeza, incertezas e estresse apresentado pelos cuidadores, que muitas vezes é agravada pela desinformação e falta de apoio psicoemocional. Nessa situação, se faz necessário orientar e proporcionar o suporte emocional a fim de favorecer um ambiente acolhedor à criança e ao familiar, visto que estes sentimentos tanto comprometem a qualidade de vida do familiar cuidador, o ato de cuidar e a melhora da criança ou do adolescente, uma vez que o cuidado dos familiares influencia na recuperação de seus filhos (Fernandes, Anjos, \& Rodrigues, 2018; Leite et al., 2020).

Todavia, o estabelecimento dessa relação entre o profissional e a família da criança com câncer, pode propiciar sentimentos ambíguos, onde, ora a família representa um elo fundamental no processo de internamento e tratamento da criança e, ora, esse profissional relata que este vínculo estabelecido pode limitar a sua atuação junto a família, sobretudo com a mulher-mãe que sofre durante todo o processo de tratamento e de possíveis situações estressantes. Nesta situação, o sentimento de medo, perda e frustração são potencializados diante de agravamento do quadro clínico e luto, que muitas vezes, são vivenciados de igual maneira pelos familiares e enfermeiros.

A situação de agravamento do quadro clínico e o processo de finitude do paciente, muitas vezes vivenciado pelo enfermeiro, caracteriza a prática da enfermagem na oncologia pediátrica como complexa e desafiadora exigindo deste profissional habilidades e competências para efetiva atuação neste cenário. Todavia, diante do inconformismo da morte de uma criança ou adolescente, se torna notório, a falta de preparo do enfermeiro em lidar com o processo de morte, acarretando o desenvolvimento de sofrimento seguido de adoecimento frente ao intenso desgaste físico e mental vivenciado no dia a dia de sua atuação (Fernandes, Anjos \& Rodrigues, 2018). Diante dessa questão, uma revisão de literatura ressalta nos estudos analisados, a fundamental necessidade de capacitação adequada para a prática assistencial e a necessidade de atualização constante em oncologia (Silva \& Bezerra, 2020).

Sobre este aspecto, pode-se observar maior número de publicações que enfatizam o acolhimento e o esclarecimento da família durante todo o processo de morte e luto, como uma forma de cuidar de crianças e adolescentes com câncer. Contudo poucos achados literários se detêm sobre o aspecto psicológico de quem cuida, neste caso, o enfermeiro, frente ao intensivo e delicado processo de cuidar do paciente com câncer independente de seu preparo e/ou formação profissional (Santos et al., 2019). No entanto, essa é uma temática ainda pouco debatida na literatura de enfermagem oncológica nacional, com discussões ainda incipientes e premente necessidade de ampliá-las, o que ratifica o desenvolvimento de estudos desta natureza.

Nesta leitura, estudo aponta que a detecção de sofrimento psíquico apresentado por estes profissionais deve ser precoce, como forma de mitigar o impacto causado na vida desse profissional e que dentre as estratégias de abrandamento psíquico destaca a indispensável capacitação que abarca a qualidade na prestação da assistência, preparo psicológico por meio do desenvolvimento de estratégias multiprofissionais, além de apoio psicoemocional (Fernandes, Anjos, \& Rodrigues, 2018).

Neste interim, a participação da equipe multidisciplinar surge como um aspecto facilitador para atuação do enfermeiro diante do tamanho desafio em trabalhar na área de oncologia pediátrica. A equipe multiprofissional é apontada pelos enfermeiros como um fator agregador, sobretudo diante da finitude, possibilitando uma forma de lidar com todas as dimensões 
do cuidar na oncologia, portanto, é um fazer multiprofissional que preconiza individualidade na atenção ao doente e seus familiares, buscando erradicar o sofrimento (Oliveira, Maranhão, \& Barroso, 2017).

Então, observa-se que a participação da equipe multidisciplinar favorece o processo de hospitalização, consequentemente possibilita a atuação do enfermeiro e demais profissionais envolvidos no cuidar a desenvolverem um plano de atendimento individualizado para cada paciente. Estudo aponta que o trabalho da equipe multidisciplinar deve ser considerado fundamental no tratamento do câncer, refletindo os avanços de diversos profissionais e o uso de múltiplas possibilidades de tratamento e apoio ao paciente. Essa ação pode ser mais bem vista no contexto dos cuidados paliativos, onde a participação da equipe multidisciplinar pode trazer grandes melhorias, como a diminuição de efeitos adversos pela evolução da doença, a redução das hospitalizações e uma melhor qualidade no final da vida (Oliveira, Maranhão, \& Barroso, 2017).

A atuação de profissionais frente aos cuidados paliativos e a terminalidade muitas vezes se apresenta como limite, pois esses fazem parte de um grupo de pessoas que vivenciam questões existenciais diárias do paciente, tornando seu trabalho um processo complexo, multifacetado, e com grandes desdobramentos emocionais (Rocha, Pereira, \& Silva, 2020). Essa situação é agravada quando esse processo de finitude envolve crianças e adolescentes, pois os enfermeiros caracterizam esse processo como abrupto por se tratar de uma interrupção precoce do ciclo natural da vida. Assim, o sentimento de perda e frustação é potencializado provocando luto nos enfermeiros, sendo evidente a falta de preparo para lidar com o processo de finitude, tornando-os suscetíveis ao sofrimento mesmo adotando estratégias defensivas, na tentativa de evitar seu próprio desgaste físico e mental, ao lidar diariamente com a possibilidade de morte de seus pacientes (Fernandes, Anjos, \& Rodrigues, 2018).

Recente estudo, associa esse sofrimento frente à terminalidade com a forma a qual as universidades estão graduando os discentes em enfermagem, pois observa-se uma lacuna considerável na formação e capacitação na área de oncologia, cuja base deve ser a graduação, visto que a maioria dos cursos de enfermagem não oferece o aprofundamento nessa área. Assim, pode-se inferir que a dicotomia existente entre a teoria e a prática decorre do ensino de enfermagem que não aborda ou aborda de forma incipiente o conhecimento em oncologia, o que implica na dificuldade e despreparo para atuar nesta área e na produção de um cuidado diferenciado (Sousa, Maracaipe, Albuquerque, \& Almeida, 2019).

Os achados de um estudo, corroboram com o presente quando afirma que para a prestação de uma assistência efetiva ao paciente, é imprescindível o preparo de todos os profissionais envolvidos no processo e destaca que o despreparo do enfermeiro na graduação pode acarretar efeitos danosos, que envolvem prejuízos psicológicos ao enfermeiro, bem como pode resultar no abandono da profissão. Ainda de acordo com o estudo, isso ocorre pela ausência de experimentação do tema morte durante a formação, levando o profissional a presumir que o cuidado efetivo ocorre apenas quando há a cura do paciente (Costa, Silva, \& Silva, 2019).

Ainda na perspectiva dos processos de finitude, os enfermeiros relataram o uso de estratégias para lidar com situações causadoras de estresse e desestabilização emocional. Nota-se que tanto os familiares, quanto a equipe de saúde sofre com a morte da criança e, dessa forma, buscam encontrar estratégias para enfrentar a perda e processar o luto. Em se tratando de cuidados paliativos, deve-se buscar o entendimento sobre as diversas manifestações de espiritualidade, como uma estratégia assertiva para aceitar o processo da morte. Sendo, a espiritualidade uma aliada tanto para o binômio paciente-família, quanto para o profissional que vivencia esse processo (Verri et al., 2019).

É importante destacar que o afastamento foi outra estratégia defensiva individual relatada pelos enfermeiros da oncologia pediátrica, como forma de separar o profissional do emocional, evitando que a assistência prestada ao paciente interfira em sua vida pessoal. Todavia, essa é uma tentativa frustrada, uma vez que o funcionamento psíquico não é divisível, ou seja, o profissional não abandona sua carga psíquica em determinado momento pré-estabelecido do dia (Viero et al., 2017). 
Questões relacionadas a instituição, como a carência de insumos e recursos materiais, também foram mencionadas pelos participantes como fator que limita sua atuação, tal fato, pode ser explicado como resultado da atual crise econômica que o país vivencia, tendo como consequência o desmonte dos serviços de saúde, sobretudo público, acarretando restrição de acesso, redução e até mesmo o fechamento de serviços, diminuição da equipe de saúde com sobrecarga de trabalho, falta de insumos para o diagnóstico e medicamentos para o tratamento, entre tantos outros aspectos. Todas estas questões, dificultam o acesso à saúde e o diagnóstico precoce, ferindo dessa forma os direitos do paciente oncológico à saúde (Oliveira, Reis, \& Silva, 2018).

Além da questão de infraestrutura, outro fator apontado como limite de atuação do enfermeiro, no que concerne à prestação dos cuidados na oncologia pediátrica foi a consciência de órgãos gestores de saúde pública no que tange a necessidade de atualização em serviço e a implementação da educação permanente voltada para a necessidade de conhecimento específico acerca da oncologia pediátrica. Tal fato é ratificado por estudos que indicam uma diferença no cuidado prestado pelo profissional que possui formação específica como, cuidados paliativos e o que não tem. Desta comparação é possível observar que os profissionais de saúde sem formação em cuidados paliativos possuem conhecimento insatisfatório em relação aos que possuem formação prévia e estes demonstram aplicar o conhecimento na prática. Alia-se ao conhecimento insuficiente o pouco investimento em educação no trabalho e na formação profissional sobre temas específicos (Cezar, Castilho, Reys, Rabin, \& Waterkemper, 2019).

Tanto os profissionais da equipe de enfermagem, quanto médicos, psicólogos, fisioterapeutas e outros profissionais envolvidos no cuidado ao paciente oncológico com indicação de cuidados paliativos, não possuem durante a sua formação, conhecimentos que abarquem o cuidado ao indivíduo portador de doenças crônicas-degenerativas fora de possibilidade de cura ou reversão da doença e os Cuidados Paliativos. Sobre esta vertente, a educação permanente se apresenta como forma de mitigar a deficiência encontrada nesta formação, além de considerada como fator potencial para a melhora do conhecimento profissional e a sua aplicação na prática, principalmente, no que se refere a segurança frente à atuação (Cezar, Castilho, Reys, Rabin, \& Waterkemper, 2019).

\section{Conclusão}

A partir dos resultados encontrados, constatou-se como possibilidade da atuação do enfermeiro, a participação da família como elo fundamental no processo de adoecimento da criança e do adolescente, bem como a participação da equipe multidisciplinar no enfrentamento da doença. Somado a isso, o desconhecimento e a falta de habilidades técnicas no campo prático da oncologia pediátrica, bem como, a lacuna do preparo psicológico, instrução da família frente ao diagnóstico, e a falta de apoio psicológico institucional frente a terminalidade e a morte, foram apontados como fatores limitadores da atuação dos enfermeiros.

Apresentar uma discussão reflexiva a respeito de limites e possibilidades de atuação do enfermeiro no contexto do câncer infanto-juvenil, se apresenta como uma importante contribuição para a prática da enfermagem em oncologia pediátrica, por oferecer aos enfermeiros novas possibilidades de atuação junto a este segmento populacional, momento decisório para a construção de vínculos afetivos com estreitamento das relações entre o paciente e sua família resultando em uma base sólida para o exercício de sua função. Desta forma, o desenvolvimento do presente estudo, se faz necessário, não apenas visando uma assistência de qualidade e diferenciada neste contexto, mas principalmente, por contribuir para uma visão ampliada, a partir do profissional, dando voz às suas emoções e sentimentos envolvidos na assistência oncológica.

Assim, torna-se premente inflar o debate quanto a necessidade de estratégias e ações que visem reduzir os fatores que limitam a atuação do enfermeiro, e, portanto, se tornam causadores de estresse e desestabilização emocional na oncologia pediátrica, tornando-o mais eficiente e equilibrado para ambas as partes envolvidas no processo de cuidado. 
Como limitação o desenvolvimento em apenas duas unidades hospitalares, sem privilegiar outros centros, que pode apresentar uma realidade diferente da encontrada neste estudo. Dessa forma, recomenda-se a realização de novos estudos para que assim seja possível contemplar os diversos desdobramentos da assistência de enfermagem oncológica.

\section{Referências}

Cezar, V. S., Castilho, R. K., Reys, K. Z., Rabin, E. G., \& Waterkemper, R. (2019). Educação Permanente em Cuidados Paliativos: uma Proposta de PesquisaAção. Revista Online de Pesquisa Cuidado é Fundamental, 11, 324-32.

Costa, E. K. C., Silva, S. B., \& Silva, J. B. (2019). O impacto do estresse na assistência do enfermeiro ao paciente terminal-revisão literária. Revista de Iniciação Científica e Extensão, 2(1), 51-56.

Fernandes, L.M.F.A., dos Anjos, L.M.F. \& Rodrigues, M.S.S. (2018) Psychological Suffering of Nurses in The Death And Dying Process of The Oncological Child. Acta de Ciência e Saúde,1(1),13-23. https://www2.1s.edu.br/actacs/index.php/ACTA/article/view/173/149

Instituto Nacional de Câncer. (2021). Tipos de Câncer. Retrieved Set 25, 2021, https://www.inca.gov.br/tipos-de-cancer/cancer-infantojuvenil.

Lacerda, C. A., de Farias Camboim, F. E., Camboim, J. C. A., Nunes, E. M., Bezerra, A. L. D., \& de Sousa, M. N. A. (2016). O lidar com a morte em unidade de terapia intensiva: dificuldades. C\&D-Revista https://www.researchgate.net/publication/318648424_O_LIDAR_COM_A_MORTE_EM_UNIDADE_DE_TERAPIA_INTENSIVA_dificuldades_relatadas_ por_enfermeiros

Leite, A. C., Pilar, T. M. I. V., Prudêncio, L. D., Sousa, A.D., Mendes, A. M., de Sousa, B. B., \& Viana, M. R. P. (2020). Atribuições do enfermeiro nos cuidados paliativos em oncologia pediátrica. Brazilian Journal of Development, 6(10), 79459-79474.

Markus, L. A, Betiolli, S. E., Souza, S. J. P., Marques, F. R. \& Migoto, M. T. (2017). The nurse's activities in patient assistance in palliative care. RGS, 17 $(1), 71-81$.

Menin, G. E \& Petterson, M. K. (2015). Terminally child life: perceptions and feelings of nurses. Rev. Bioética, 23(3),608-614

Minayo, M. C. S. (2014). O desafio do conhecimento: pesquisa qualitativa em saúde. (14 ed) São Paulo. Hucitec

Oliveira, J. M, Reis, J. B \& Silva, R. A. (2018). Search for oncological care: perception of patients and family members. Rev enferm UFPE on line,12(4),93846.

Oliveira, T. C. B, Maranhão, T. L. G \& Barroso, M. L. (2017). Multiprofessional Pediatric Oncology Palliative Care Team: A Systematic Review. Rev. Psic. 11(35), 492-530. https://idonline.emnuvens.com.br/id/article/view/754/1061

Paula, D. P. S., Silva, G. R. C., Andrade, J. M. O. \& Paraiso, A. F. (2019) Câncer infantojuvenil do âmbito familiar: percepções e experiências frente ao diagnóstico. Rev Cuid, 10(1), e570

Rocha, R. C. N. P., Pereira, E. R., \& Silva, R. M. C. R. A. (2020) The meaning of life of nurses working in palliative care: integrative literature review, 22:112 .

Rodrigues, J. R. G., Siqueira Junior, A. C. \& Siqueira, F. P. C. (2020) Nursing consultation in pediatric oncology: a tool for empowering parentes. Rev.pesq.: cuid. Fundam, 12,211-221

Santana, M. E., Costa, E. G. O., Corrêa, A. R. S. \& Ximenes, W.L.O. (2017). O cuidar em oncologia pediátrica: um estudo baseado no processo de enfermagem. Revista Destaques Acadêmicos, 9(3),228-236.

Santos, S. R., Bispo, T. F., Morais, R. X. S., Amorim, M. N. S., Costa, C. S. C. \& Siqueira, S. C. (2019) The acting of nurses in palliative care for children with cancer. RRS-FESGO, 2(3),61-64. http://revistaadmmade.estacio.br/index.php/rrsfesgo/article/viewFile/7183/47966155

Silva, G. F., de Assis, M. T. B., \& Pinto, N. B. F. (2021). Cuidados Paliativos na Criança com Câncer: o papel do enfermeiro na assistência do cuidar. Brazilian Journal of Development, 7(5), 53524-53540.

Silva, M. F., \& Bezerra, M. L. R. (2020). Atuação do enfermeiro no atendimento aos cuidados continuados na oncologia. Revista JRG de Estudos Acadêmicos, 3(6), 123-137.

Sousa, G. S. B., Maracaipe, L. F, Albuquerque, I. A \& Almeida, A. B. (2019) Training process of the nurse in onco-pediatricpractice. Rev Inic Cient Ext., 2(1), 46-50. https://revistasfacesa.senaaires.com.br/index.php/iniciacaocientifica/article/view/141/96

Souza, M. A. R. D., Wall, M. L., Thuler, A. C. D. M., Lowen, I. M. V. \& Peres, A. M. (2018). The use of IRAMUTEQ software for data analysis in qualitative research. Revista da Escola de Enfermagem da USP, 52, e03353

Souza, R. S., Araújo, F. L. D., Manzo, B. F., Marcatto, J. D. O., Montenegro, L. C., Silva, P. R. M., \& Simão, D. A. D. S. (2020). Care in pediatric oncology: a cross-sectional analysis of the quality of life of nursing professionals. Revista Brasileira de Enfermagem,73(Suppl 6):e20190639. http://dx.doi.org/10.1590/0034-7167-2019-0639

Verri, E. R., Santana Bitencourt, N. A., da Silva Oliveira, J. A., dos Santos Júnior, R., Silva Marques, H., Alves Porto, M., \& Rodrigues, D. G. (2019). Nursing Professionals: Understanding About Pediatric Palliative Care. Journal of Nursing UFPE/Revista de Enfermagem UFPE, 13(1), 126-136

Viero V., Beck, C. L. C., Coelho, A. P. F, Pai, D. D., Freitas, P. H. \& Fernandes, M. N. S. (2017) Pediatric oncology nursing workers: the use of defensive strategies at work. Esc Anna Nery,21(4), e20170058. https://www.redalyc.org/pdf/1277/127752022018.pdf 